\title{
Solubility of Solids in Supercritical Fluids: The Mendez-Santiago Teja Model Revisited
}

\author{
Loubna Nasri ${ }^{*}$, Zouhir Bensetiti ${ }^{2}$ \\ ${ }^{1}$ Departement of Pharmaceutical Engineering, University of Constantine 3, Constantine, Algeria \\ ${ }^{2}$ Unisignal Inc., Brossard, Canada \\ Email: ${ }^{*}$ loubnanasri@yahoo.com
}

Received 4 July 2015; accepted 25 July 2015; published 29 July 2015

Copyright (C) 2015 by authors and Scientific Research Publishing Inc.

This work is licensed under the Creative Commons Attribution International License (CC BY).

http://creativecommons.org/licenses/by/4.0/

(c) (1) 0 ipen Access

\begin{abstract}
For the development and enhancement of supercritical applications, it is crucial to know the solubility of the considered compound in the supercritical fluid (SCF) in order to determine the conditions to achieve the best outcome. Many models have been developed to calculate supercritical solubility behavior and most can be either a semi empirical relationship or based on an equation of state. The Mendez-Santiago and Teja (MST) model is one of the most semiempirical models used. In this work, a review of the (MST) model from an accuracy and ability viewpoint is accomplished to give concise discussion and so to the proposition of a modification.
\end{abstract}

\section{Keywords}

\section{Supercritical Fluids, Solubility, Dilute Solutions, MST Model}

\section{Introduction}

Supercritical-Fluid Extraction (SCFE) is a separation method for which increased interest is shown for cases where traditional separation methods such as distillation have become too expensive, or cannot be applied. The advantages of SCFE are well documented and several commercial applications have been reported [1]. Supercritical fluids are environmentally benign solvents; their properties that are interesting from an extraction viewpoint include the diffusion coefficient, the density and the viscosity. The design and the development of SCFE processes depend on the ability to model and predict accurately the solid-supercritical fluid equilibria. Many of the existing simple predictive models are not sufficiently accurate to apply in the design and development of these processes [2]. Even the more sophisticated models are subject to serious errors when they are used for calculations near critical points, an area of great interest in terms of supercritical extraction. An additional com-

${ }^{*}$ Corresponding author. 
plication is that many of the solute molecules of interest are large and polar, while the solvent molecules such as carbon dioxide tend to be small and of low polarity. This makes the thermodynamics less amenable to the usual modeling methods [3].

\section{Background}

Different models have been used to predict the solubility of solids in SCFs, such as theoretical equations of state or semiempirical equations. Theoretical models like cubic equation of state or perturbed equations need large and complicated computational methods and the knowledge of the solid properties [4]. The problem starts with the availability of these properties, acentric factor and critical properties cannot be determined experimentally in most cases, so they should be evaluated using predictive methods, like group contributions (GC). Different approaches are proposed in the literature, but it is quite common to encounter molecules for which the methods are not applicable [5]. The same situation occurs with the sublimation pressure which plays a dominant role in the correlation of solubility data. In many cases, this quantity cannot be measured since its value is below the limit of any experimental method. Also for this property, GC methods have been suggested in the literature, but one has no way of controlling the validity of the calculated value [5]. Garnier et al. [6] stated that it was shown in the literature that the error in sublimation, which is very low for high molecular weight compounds, is in many cases responsible for large deviations between experimental and calculated solubilities. On the other hand, semiempirical equations like density based models do not need solid properties. They are based on simple error minimization and they use only available independent variables like pressure, temperature and density of pure solvent. The only drawback is the semiempirical character, which means that the solubility data are needed [4]. Recently, attempts have been reported to minimize the deficiency in prediction accuracy resulting from the error of sublimation pressures for non-volatile components that are used for the prediction of solubility solids in SFs [6] [7]. Among them we focus on the attempt of Mendez-Santiago and Teja [7] where they developed a simplified correlation to solid solubility data based on the theory of dilute solutions that was used earlier for the same purpose by Harvey [8]. In case of unavailability of sublimation pressure they have incorporated a ClausiusClapeyron-type expression and derived a modified relation for the solid solubility.

In a previous work [9], we have presented a methodology for the correlation and prediction of the solubility of some solids with different functional groups in different supercritical fluids. The methodology is based on the expanded liquid model theory which does not require the knowledge of the solute critical properties and sublimation pressure. For the comparison of results we have considered some literature models that account for effect of the system conditions in addition to the physical properties as sublimation pressure of the solute through their introduction of the enhancement factor such as those of Mendez-Santiago and Teja (MST). The results obtained show that for many of the 33 binary systems considered, the "modified" model of Mendez-Santiago and Teja gives better results (in term of average differences) than the original one. This was very surprising and motivates us to revisit the two models.

\section{Solubility Correlation}

Correlating the solubility to the fluid density is a common approach for modeling solid solubilities in SCF's. Several authors have noticed that the logarithm of the solubility is a linear function of the density or sometimes of the logarithm of the density. The linearity of plots on these coordinates was first explained by Kumar and Johnston [10] in a derivation limited to the critical isotherms and they showed that this type of correlation is valid in the reduced density interval $0.5 \leq \rho_{r} \leq 2.0$ where $\rho_{r} \equiv \rho / \rho_{c}$. A more general derivation was given by Harvey [10] who adapted the expression of Levelt Sengers [11] who has shown that near the critical point of the solvent, the expression for Henry's constant can be simplified and may be written as:

$$
T \ln \left(K / f_{1}\right)=A+B\left(\rho-\rho_{c}\right)
$$

where $T$ is the temperature, $K$ is Henry's constant, $f_{1}$ and $\rho$ are the fugacity and density of the solvent at saturation, $\rho_{c}$ is the critical density of the solvent, and $A$ and $B$ are constants. Harvey [8] worked with an effective Henry's constant defined by

$$
K_{H}^{\text {eff }}=f_{2} / y_{2}
$$

where $y_{2}$ is the mole fraction solubility, $f_{2}$ is the solute fugacity, which is fixed by the presence of an equili- 
brium solid phase and is proportional to the vapor pressure of the pure solid as follows:

$$
f_{2}=P_{2}^{s} \exp \left[V_{2}\left(P-P_{2}^{s}\right) / R T\right]
$$

where $P_{2}^{s}$ and $V_{2}$ are the sublimation pressure and the molar volume of the solid. By substituting the fugacity in Equation (2) the effective Henry's constant become:

$$
K_{H}^{\text {eff }}=P_{2}^{s} \exp \left[V_{2}\left(P-P_{2}^{s}\right) / R T\right] / y_{2}
$$

Harvey [8] combined expression (1) and (4) to obtain:

$$
T \ln (E)=-\left[A+B\left(\rho-\rho_{c}\right)\right]+V_{2}\left(P-P_{2}^{s}\right) / R-T \ln \varnothing_{1}
$$

where $E$ is the enhancement factor $\left(E=y_{2} P / P_{2}^{s}\right)$, and $\varnothing_{1}$ is the fugacity coefficient of the solvent. By neglecting terms, Mendez-Santiago and Teja [7] simplified Equation (5) to obtain:

$$
T \ln E=-\left[A+B\left(\rho-\rho_{c}\right)\right] \equiv A_{1}+B_{1} \rho
$$

Application of Equation (6) requires the sublimation pressure of the solute which is not available as stated in Section 2. Mendez-Santiago and Teja [7] circumvented this difficulty by the incorporation of a Clausius-Clapeyron type expression and derived an equation with three adjustable parameters as below:

$$
T \ln \left(y_{2} P\right)=A^{\prime}+B^{\prime} \rho+C^{\prime} T
$$

Correlation capability of Equations (6) and (7) are presented by Mendez-Santiago and Teja [7] in terms of absolute average difference $A A D \%$ between experimental and calculated solubilities:

$$
A A D \%=\frac{100}{N} \sum_{1}^{N} \frac{\left|y_{2(\exp )}-y_{2(\text { cal })}\right|}{y_{2(\exp )}},(N \text { number of data points) }
$$

At this point, reader can think obviously that if we have a common solute for which sublimation pressures are available and if these data are well represented by a Clausius-Clapeyron expression, Equation (6) and (7) must give the same $A A D \%$ (in the same range of T, $\mathrm{P}$ and for the same solubility data). But this is not the case, Mendez-Santiago and Teja [7] utilized Equations (6) and (7) for the same system solid-SC carbon dioxide just for two solutes: eicosanoic acid and myristic acid, and there is a difference of $33 \%$ between the $A A D \%$ of the two correlations. Also, previous results [9] show considerable differences between correlation utilizing Equations (6) and (7) for many systems among 33 systems solute-SCF considered. In fitting data, it's known that increasing the number of model parameter will generally improve the correlation results, but in the case of Equation (7) the third parameter is not an additional one but it follows from the Clausius-Clapeyron expression.

From another point of view, to check the consistence and ability of Equations (6) and (7) we have reconsidered them for some systems presented in Table 1 as follows:

$$
T \ln E=-A-B\left(\rho-\rho_{c}\right)
$$

Using dimensionless equations is very useful as stated by Sparks et al. [12], so by introducing reduced coordinates of solvent Equation (6) become:

$$
T_{r} \ln E=\alpha+\beta\left(\rho_{r}-1\right)
$$

where $\alpha=\frac{-A}{T_{c}}$ and $\beta=\frac{B \rho_{c}}{T_{c}}$.

When sublimation pressure is not available, Equation (10) is decomposed as below:

$$
\begin{gathered}
T_{r}\left(\ln y_{2} P-\ln P_{2}^{s}\right)=\alpha+\beta\left(\rho_{r}-1\right) \\
T_{r} \ln y_{2} P_{r}=\alpha+\beta\left(\rho_{r}-1\right)-T_{r} \ln P_{c}+T_{r} \ln P_{2}^{s}
\end{gathered}
$$

A Clausius-Clapeyron-type expression for the sublimation pressure $\left(\ln P_{2}^{s}=A^{s}-\frac{\Delta H^{s}}{R T}\right)$ [13] is introduced 
Table 1. Considered systems.

\begin{tabular}{|c|c|c|c|}
\hline Binary systems & $\mathrm{N}$ & $\rho_{\mathrm{r}}$ range & References \\
\hline Naphthalene- $\mathrm{CO}_{2}$ & 242 & $0.51-1.98$ & L1 - L17 \\
\hline Pyrene- $\mathrm{CO}_{2}$ & 235 & $0.78-1.99$ & L18, L19, L20, L25 \\
\hline Naphthalene-ethylene & 166 & $0.52-1.99$ & L1, L6, L42, L43 \\
\hline Naphthalene-ethane & 48 & $0.56-1.99$ & L22, L40 \\
\hline Anthracene-ethane & 13 & $1.49-1.99$ & L22 \\
\hline Anthracene-ethylene & 27 & $0.75-1.97$ & $\mathrm{~L} 40$ \\
\hline Biphenyl- $\mathrm{CO}_{2}$ & 57 & $0.55-1.99$ & L9, L5, L12 \\
\hline Biphenyl-ethane & 8 & $1.32-1.94$ & $\mathrm{~L} 22$ \\
\hline 2,3-dimethylnaphtalene-ethylene & 18 & $0.64-1.93$ & L21 \\
\hline 2,7-dimethylnaphtalene- $\mathrm{CO}_{2}$ & 10 & $0.69-1.91$ & L8 \\
\hline Fluoranthene- $\mathrm{CO}_{2}$ & 68 & $0.62-1.99$ & L27, L24 \\
\hline Phenanthrene-ethylene & 42 & $0.45-1.49$ & L40, L21, L19 \\
\hline Triphenylene- $\mathrm{CO}_{2}$ & 53 & $0.83-1.99$ & L27, L25 \\
\hline lauric acid- $\mathrm{CO}_{2}$ & 24 & $0.53-1.94$ & L33, L34, L35 \\
\hline stearic acid- $\mathrm{CO}_{2}$ & 28 & $0.94-1.91$ & L37, L41, L38, L39 \\
\hline Phenol- $\mathrm{CO}_{2}$ & 73 & $0.51-1.91$ & L31, L32, \\
\hline 1-naphtol- $\mathrm{CO}_{2}$ & 64 & $0.54-1.98$ & L26, L36 \\
\hline 2-naphtol- $\mathrm{CO}_{2}$ & 48 & $0.65-1.99$ & L22, L29, L44 \\
\hline Acridine-ethane & 26 & $0.51-1.99$ & L22 \\
\hline 2-aminofluorene- $\mathrm{CO}_{2}$ & 15 & $0.70-1.97$ & L22 \\
\hline 9,10-anthraquinone- $\mathrm{CO}_{2}$ & 16 & $0.71-1.98$ & L28 \\
\hline benzoic acid- $\mathrm{CO}_{2}$ & 75 & $0.64-1.97$ & L22, L29, L23, L21, L30 \\
\hline benzoic acid-ethane & 23 & $0.56-1.98$ & $\mathrm{~L} 22$ \\
\hline
\end{tabular}

to apply Equation (12), ( $A^{s}$ is a constant and $\Delta H^{s}$ is the sublimation enthalpy):

$$
\begin{gathered}
T_{r} \ln y_{2} P_{r}=\left(\alpha-\frac{\Delta H^{s}}{R T_{C}}\right)+\beta\left(\rho_{r}-1\right)+\left(A^{s}-\ln P_{C}\right) T_{r} \\
T_{r} \ln y_{2} P_{r}=\alpha^{\prime}+\beta^{\prime}\left(\rho_{r}-1\right)+\gamma^{\prime} T_{r}
\end{gathered}
$$

From Table 2, if we note by $\left(\alpha^{\prime}\right)_{\text {cal }}$ the parameter $\alpha^{\prime}$ calculated with physical properties from Table 3 and

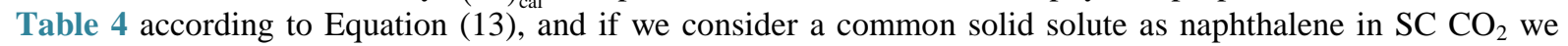
found:

$$
\left(\alpha^{\prime}\right)_{\text {cal }}=-6.7-\frac{72300}{8.314 \times 304.14}=-35.30
$$

And the parameter $\alpha^{\prime}$ obtained by regression of data by Equation (14), $\alpha^{\prime}=-30.63$.

The difference between the two values of $\left(\alpha^{\prime}\right)$ is very important, and the same remark is obtained for the others binary systems solid-SC fluid presented in Table 2. These systems are some of those for which the difference between correlation by Equations (10) and (14) is considerable (Figure 1), and all solubility data are in the range $(0.5-2)$ of reduced density (Table 1$)$ as stated in Section 3. 
Table 2. Regression parameters and average deviations.

\begin{tabular}{|c|c|c|c|c|c|c|c|c|c|}
\hline System & $N$ & $\alpha$ & $\beta$ & $\alpha^{\prime}\left(\alpha_{m}\right)$ & $\beta^{\prime}\left(\beta_{m}\right)$ & $\gamma^{\prime}\left(\gamma_{m}\right)$ & $\left(\alpha^{\prime}\right)_{c a l}$ & $\left(\alpha^{\prime \prime}\right)_{c a l}$ & $A A D \%$ \\
\hline Acridine-ethane & 26 & & & & & & & & \\
\hline Equation (10) & & 8.91 & 4.63 & & & & & & 26.20 \\
\hline Equation (14) & & & & -31.09 & -4.8 & 23.99 & & & 20.74 \\
\hline Equation (15) & & & & $(4.75)$ & $(4.8)$ & $(3.82)$ & -27.14 & -31.33 & 20.74 \\
\hline Anthracene-ethylene & 27 & & & & & & & & \\
\hline Equation (10) & & 11.55 & 5.22 & & & & & & 14.3 \\
\hline Equation (14) & & & & -26.90 & -5.33 & 16.82 & & & 7.64 \\
\hline Equation (15) & & & & (17.08) & (5.33) & $(-4.48)$ & -32.15 & -26.59 & 7.66 \\
\hline Anthracene-ethane & 13 & & & & & & & & \\
\hline Equation (10) & & 10.4 & 4.98 & & & & & & 7.66 \\
\hline Equation (14) & & & & -28.14 & -4.91 & 19.40 & & & 5.82 \\
\hline Equation (15) & & & & (12.61) & $(4.77)$ & $(-1.86)$ & -30.05 & -27.81 & 5.78 \\
\hline Benzoic acid- $\mathrm{CO}_{2}$ & 75 & & & & & & & & \\
\hline Equation (10) & & 8.15 & 4.93 & & & & & & 11.40 \\
\hline Equation (14) & & & & -29.95 & -5.08 & 24.56 & & & 9.71 \\
\hline Equation (15) & & & & $(4.98)$ & $(5.08)$ & $(2.92)$ & -26.93 & -30.10 & 9.71 \\
\hline Benzoic acid-ethane & 23 & & & & & & & & \\
\hline Equation (10) & & 6.63 & 5.16 & & & & & & 16.11 \\
\hline Equation (14) & & & & -31.79 & -5.33 & 24.95 & & & 11.57 \\
\hline Equation (15) & & & & $(3.10)$ & $(5.32)$ & (3.24) & -28.31 & -31.84 & 11.56 \\
\hline biphenyl- $\mathrm{CO}_{2}$ & 62 & & & & & & & & \\
\hline Equation (10) & & 7.89 & 4.09 & & & & & & 19.8 \\
\hline Equation (14) & & & & -31.77 & 4.22 & 24.51 & & & 15.5 \\
\hline Equation (15) & & & & $(1.25)$ & $(4.22)$ & $(6.28)$ & -25.09 & -31.73 & 15.5 \\
\hline biphenyl-ethane & 8 & & & & & & & & \\
\hline Equation (10) & & 7.53 & 4.42 & & & & & & 9.81 \\
\hline Equation (14) & & & & -34.92 & 5.02 & 27.55 & & & 5.67 \\
\hline Equation (15) & & & & $(-0.51)$ & $(4.96)$ & $(7.45)$ & -25.32 & -33.94 & 5.60 \\
\hline 2,3-Dimethylnaphtalene-ethylene & 18 & & & & & & & & \\
\hline Equation (10) & & 8.54 & 5.72 & & & & & & 16.78 \\
\hline Equation (14) & & & & -35.04 & 6.41 & 24.14 & & & 14.62 \\
\hline Equation (15) & & & & $(1.22)$ & $(6.32)$ & $(6.19)$ & -26.08 & -33.98 & 14.70 \\
\hline 2,7-Dimethylnaphtalène- $\mathrm{CO}_{2}$ & 10 & & & & & & & & \\
\hline Equation (10) & & 8.32 & 3.44 & & & & & & 8.52 \\
\hline Equation (14) & & & & -26.67 & -3.63 & 23.35 & & & 6.73 \\
\hline Equation (15) & & & & (5.94) & (3.76) & (2.03) & - & - & 6.43 \\
\hline
\end{tabular}




\section{Continued}

Fluoranthene- $\mathrm{CO}_{2}$

Equation (10)

Equation (14)

Equation (15)

Lauric acid- $\mathrm{CO}_{2}$

Equation (10)

Equation (14)

Equation (15)

Naphtalene- $\mathrm{CO}_{2}$

Equation (10)

Equation (14)

Equation (15)

Naphthalene-ethylene

Equation (10)

Equation (14)

Equation (15)

Naphthalene-ethane

Equation (10)

Equation (14)

Equation (15)

1-naphtol- $\mathrm{CO}_{2}$

Equation (10)

Equation (14)

Equation (15)

2-naphtol- $\mathrm{CO}_{2}$

Equation (10)

Equation (14)

Equation (15)

Phenanthrene-ethylene

Equation (10)

Equation (14)

Equation (15)

Phenol- $\mathrm{CO}_{2}$

Equation (10)

Equation (14)

Equation (15)
68

$11.15 \quad 4.42$

$\begin{array}{rrrrrr}-24.80 & 4.28 & 18.31 & & & 11.21 \\ (15.76) & (4.28) & (-4.25) & -29.35 & -24.74 & 11.21\end{array}$

24

$11.2 \quad 6.83$

$\begin{array}{llll}-30.01 & 6.65 & 21.02 & 11.10\end{array}$

$\begin{array}{llllll}(23.19) & (6.67) & (-11.68) & -41.21 & -29.35 & 11.22\end{array}$

242

$-6.7-3.31$

$\begin{array}{lccccc}-30.63 & 3.68 & 28.36 & & & 11.79 \\ (-1.7) & (3.68) & (7.96) & -35.30 & -30.30 & 11.79\end{array}$

167

$7.42 \quad 4.18$

$\begin{array}{lll}-28.39 & 4.54 \quad 20.65\end{array}$

16.02

(2.80) (4.54)

(4.15)

$\begin{array}{ll}-23.38 & -28.00\end{array}$

11.28

11.28

48

$6.36 \quad 3.70$

$-28.41 \quad 3.85 \quad 22.23$

15.19

(0.43) (3.85)

(5.72)

12.47

12.47

64

$7.84 \quad 3.83$

$$
\begin{array}{lll}
-31.21 & -3.86 \quad 25.42
\end{array}
$$

20.41

(2.5) (3.91)

(5.03)

$-27.39-32.14$

17.99

48

$8.24 \quad 4.53$

$\begin{array}{lll}-26.2 & -4.30 \quad 20.03\end{array}$

14.08

(11.08)

(4.3)

$(-2.57)$

$-29.02$

$-26.18$

11.91

42

$4.08 \quad 5.83$

$\begin{array}{lll}-31.68 & 5.64 \quad 15.03\end{array}$

12.55

(13.33)

(5.56)

$(-2.87)$

$-36.8$

9.52

69

$5.52 \quad 2.99$

$\begin{array}{lll}-30.76 & -3.43 \quad 28.71\end{array}$

25.65

$(-3.97)$

(3.43)

(9.01)

$-21.29$

$-30.78$ 


\section{Continued}

\begin{tabular}{|c|c|c|c|c|c|c|c|c|c|}
\hline Pyrene- $\mathrm{CO}_{2}$ & 235 & & & & & & & & \\
\hline Equation (10) & & 10.55 & 4.89 & & & & & & 9.60 \\
\hline Equation (14) & & & & -26.88 & -4.79 & 19.22 & & & 6.41 \\
\hline Equation (15) & & & & (13.90) & $(4.79)$ & $(-3.13)$ & -29.31 & -25.97 & 6.41 \\
\hline Stearic acid- $\mathrm{CO}_{2}$ & 28 & & & & & & & & \\
\hline Equation (10) & & 15.14 & 4.58 & & & & & & 8.80 \\
\hline Equation (14) & & & & -48.74 & -5.16 & 40.02 & & & 7.53 \\
\hline Equation (15) & & & & (12.44) & $(5.04)$ & $(2.30)$ & -47.01 & -50.04 & 7.20 \\
\hline Triphenylene- $\mathrm{CO}_{2}$ & 53 & & & & & & & & \\
\hline Equation (10) & & 13.1 & 5.16 & & & & & & 12.84 \\
\hline Equation (14) & & & & -27.86 & -5.03 & 17.76 & & & 4.77 \\
\hline Equation (15) & & & & (19.05) & $(4.98)$ & $(-5.58)$ & -32.17 & -26.90 & 5.02 \\
\hline 9,10-Anthraquinone- $\mathrm{CO}_{2}$ & 16 & & & & & & & & \\
\hline Equation (10) & & 10.80 & 6.53 & & & & & & 10.01 \\
\hline Equation (14) & & & & -35.76 & -6.69 & 24.63 & & & 7.36 \\
\hline Equation (15) & & & & $(5.81)$ & (6.69) & $(4.73)$ & -31.88 & -36.06 & 7.40 \\
\hline 2-Aminofluorene- $\mathrm{CO}_{2}$ & 15 & & & & & & & & \\
\hline Equation (10) & & 9.92 & 5.20 & & & & & & 24.48 \\
\hline Equation (14) & & & & -38.53 & 5.56 & 24.74 & & & 16.84 \\
\hline Equation (15) & & & & $(2.76)$ & (5.55) & $(6.44)$ & -33.08 & -40.09 & 16.84 \\
\hline
\end{tabular}

[ Equation (10) $\quad$ Equation (14) Equation (15)

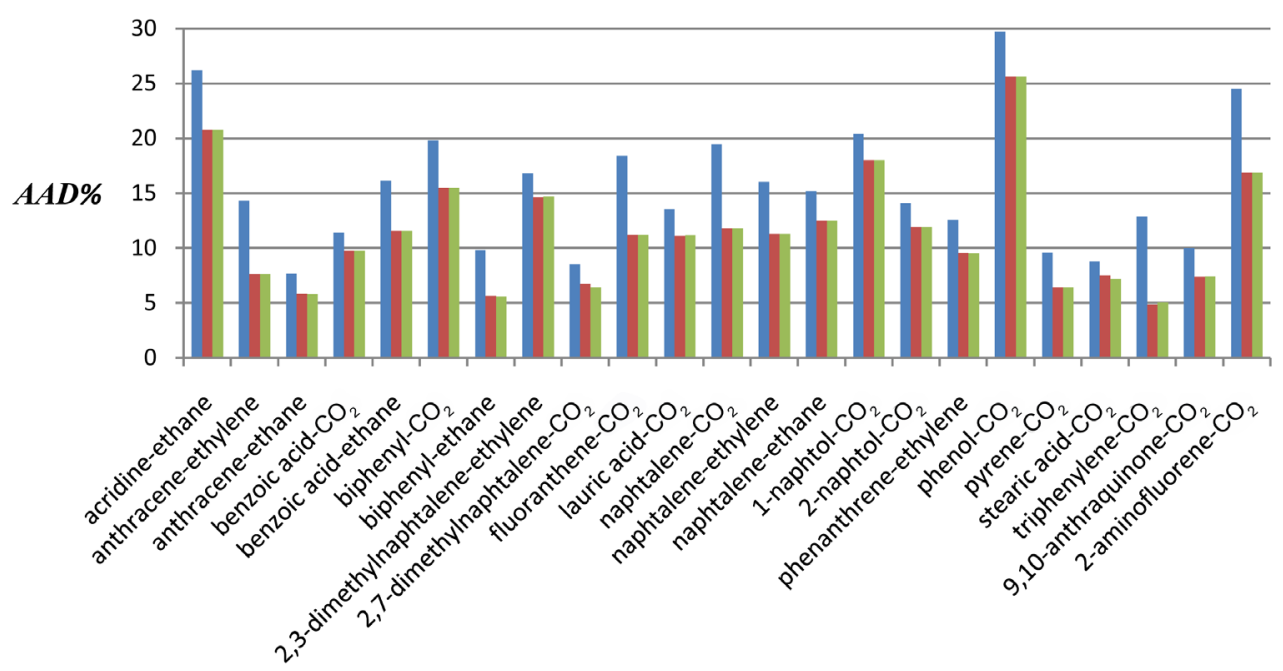

Figure 1. Histogram comparison of the different average differences.

Table 3. Solvents physical properties.

\begin{tabular}{cccc}
\hline Solvent & $T_{c}(\mathrm{~K})$ & $P_{c}(\mathrm{bar})$ & $\rho_{c}\left(\mathrm{~mol} / \mathrm{cm}^{3}\right) \times 100$ \\
\hline $\mathrm{CO}_{2}$ & 304.2 & 73.8 & 1.063 \\
Ethane & 305.3 & 48.8 & 0.687 \\
Ethylene & 382.3 & 50.4 & 0.764 \\
\hline
\end{tabular}

afrom reference [14]. 
Table 4. Coefficients for sublimation pressure estimation and sublimation enthalpies of considered solids.

\begin{tabular}{|c|c|c|c|c|c|c|}
\hline \multicolumn{7}{|c|}{$P^{S}(\mathrm{~Pa})=10^{\left(A-\frac{B}{T}\right)}$} \\
\hline Solute & $A$ & $B$ & $T$ range $(\mathrm{K})$ & Ref & $\Delta H^{s} \quad(\mathrm{~kJ} / \mathrm{mol})$ & Ref \\
\hline Acridine & 13.721 & 4740.1 & $308-343$ & [15] & 91.6 & [16] \\
\hline Anthracene & 14.275 & 5401.0 & $303-373$ & [17] & 102.6 & [18] \\
\hline 2-Aminofluorene & 14.865 & 5469.0 & $308-343$ & [15] & 110.4 & [19] \\
\hline 9,10-Anthraquinone & 14.05 & 5618.9 & $308-318$ & [20] & 107.9 & [21] \\
\hline Biphenyl & 14.804 & 4367.4 & $308-343$ & [15] & 83.4 & [22] \\
\hline Benzoic acid & 14.408 & 4618.1 & $308-343$ & [15] & 88.7 & [23] \\
\hline 2,3-Dimethylnaphtalene & 14.065 & 4302.5 & $308-328$ & [24] & 82.2 & [25] \\
\hline 2,7-Dimethylnaphtalene & 14.464 & 4386.7 & $308-328$ & {$[20]$} & na & - \\
\hline Fluoranthene & 14.795 & 5357.0 & $298-358$ & [17] & 102.4 & [26] \\
\hline Lauric acid & 22.022 & 7322.0 & $295-314$ & [27] & 132.6 & [28] \\
\hline Naphthalene & 13.865 & 3823.1 & $250-340$ & {$[27]^{a}$} & 72.3 & [29] \\
\hline 1-Naphtol & 10.683 & 3148.9 & $308-328$ & [15] & 89.1 & [30] \\
\hline 2-Naphtol & 14.815 & 4923.9 & $308-343$ & [15] & 94.2 & [31] \\
\hline Phenanthrene & 14.343 & 4776.7 & $300-360$ & {$[27]^{a}$} & 95.0 & [17] \\
\hline Phenol & 13.689 & 3586.4 & $309-333$ & [32] & 67.8 & [33] \\
\hline Pyrene & 13.395 & 4904.0 & $308-398$ & [34] & 100.8 & [35] \\
\hline Stearic acid & 21.021 & 7957.0 & $308-338$ & {$[36]$} & 158.0 & [37] \\
\hline Triphenylene & 14.462 & 5804.1 & $300-340$ & [32] & 114.5 & [38] \\
\hline
\end{tabular}

${ }^{\mathrm{a}}$ Data interpolated in this work, na/not available.

\section{Table 5. References of solubility data.}

L1: Russian journal of physical chemistry 1964; 38: 9

L2: J. Supercritical Fluids. 1996; 9: 3

L3: J. Chem. Eng. Data 1998; 43: 400-402

L4: J. Chem. Eng. Data 1999; 48: 951-957

L5: J. Chem. Eng. Data 1980; 25, 4, 326-329

L6: J. Supercritical. Fluids 1988; 1: 1

L7: J. Chem. Eng. Data 1989, 36: 4, 430-432

L8: J. Chem. Eng. Data 1993, 38, 3

L9: Fluid Phase Equilibria 1992; 81:321-341

L10: Fluid Phase Equilibria 1995, 107, 189-200

L11: Ind. Eng. Chem. Res. 2000, 39, 4609-4614

L12: J. Supercritical. Fluids 1995, 8, 1, 15-19

L13: J. chem. Eng. Data 1988, 33, 1 , 35-37

L14: J. Chem. Eng. Data 1985, 30, 1

L15: J. Physical Chemistry 1986, 90, 17

L16: J. Chem. Eng. Data 2000, 45: 464-466

L17: J. Chem. Eng. Data 2000, 45: 358-361

L18: J. Chem. Eng. Data 1990, 35: 355-360

L19: Ind. Eng. Chem. Fundamentals 1982, 21, 3

L20: J. Supercritical Fluids 1997, 10, 175-189

L21: J. Chem. Eng. Data 1981, 26, 1, 47-51

L22: J. Chem. Eng. Data 1986, 31, 2, 204-212
L23: J. Chem. Eng. Data 2001, 46, 5, 1156-1159

L24: J. Chem. Eng. Data 1996, 41, 1466-1469

L25: Ind. Eng. Chem. Res 1995, 34, 340-346

L26: J. Chem. Eng. Data 1995, 40, 953-858

L27: J. Chem. Eng. Data 2000, 45, 53

L28: J. Chem. Eng. Data 1997, 42, 463-466

L29: Ind. Eng. Chem. Res 26, 1, 1987, 56-65

L30: Fluid Phase Equilibria 2004, 226, 9-13

L31: J. Chem. Eng. Data 1980, 25, 257-259

L32: Hwahak Konghak Journal 1993, 31, 6, 637

L33: J. Chem. Eng. Data 1988, 33, 3, 327-333

L34: J. Chem. Eng. Data 2008, 53, vol. 11

L35: J. Am. Oil. Chem. Soc. 1992, 69, p. 1069

L36: Fluid Phase Equilibria 1987, 34, 37-47

L37: J. Chem. Eng. Data 1993, 38, 506-508

L38: J. Chem. Eng. Data 2008, 53, 2913-2917

L39: J. Chem. Thermodynamics 2010, 42, 193-197

L40: AIChE journal 1981, 27, No 5, 773-779

L41: J. Chem. Eng. Data 1989, 34, 184-187

L42: J. Amer. Chemic. Socie 1953, 57, 575-578

L43: J. Amer. Chemic. Socie 1948, 70, 4085-4089

L44: Fluid Phase Equilibria 2003, 207, 183-192 
The last point is based on the step of neglecting terms in Equation (5) which has not been argued by MendezSantiago and Teja [7] and some authors as Hansen et al. [39] stated that it was even a surprising step. So from the previous remarks, we can think that the neglected term (especially related to temperature) is for many systems solid-SCF non negligible.

For all these points, we opted for a modification to Equation (10) by adding a "correction" term proportional to the temperature without changing the original form as follows:

$$
T_{r} \ln E=\alpha_{m}+\beta_{m}\left(\rho_{r}-1\right)+\gamma_{m} T_{r}
$$

Subscript $(m)$ is for the modification, and by introducing a cluasius-clapeyron expression for sublimation pressure, we can write:

$$
T_{r} \ln y_{2} P_{r}=\left(\alpha_{m}-\frac{\Delta H^{s}}{R T_{C}}\right)+\beta_{m}\left(\rho_{r}-1\right)+\left(\gamma_{m}+A^{s}-\ln P_{C}\right) T_{r}
$$

Equation (16) can be presented as follows:

$$
T_{r} \ln y_{2} P_{r}=\alpha^{\prime \prime}+\beta^{\prime \prime}\left(\rho_{r}-1\right)+\gamma^{\prime \prime} T_{r}
$$

Thus, Equation (17) has the same form of Equation (14) but just with different parameters.

From Table 2 and Figure 1, we can see clearly that with the opted modification:

1) Correlation of data using Equation (15) and Equation (14) yield the same $A A D \%$;

2) And if we note by $\left(\alpha^{\prime \prime}\right)_{c a l}$ the coefficient calculated by Equation (15) and physical properties

$$
\left[\left(\alpha^{\prime \prime}\right)_{\text {cal }}=\left(\alpha_{m}-\frac{\Delta H^{s}}{R T_{C}}\right)\right],\left(\alpha^{\prime \prime}\right)_{\text {cal }} \text { is very close to the regression parameter } \alpha^{\prime} \text {. }
$$

\section{Conclusion}

This paper focuses on the application of the Mendez-Santiago Teja (MST) model for the correlation of solubility of solids in different supercritical fluids. Attention is paid to the presentation of the different limitations of the model. Analysis and results reported in this work show that a modification of the MST model is necessary. The modification proposed herewith keeps the simple form of original equations and is successful for all systems considered.

\section{References}

[1] Coutsikos, P., Magoulas, K. and Kontogeorgis, G.M. (2003) Prediction of Solid-Gas Equilibria with the Peng-Robinson Equation of State. The Journal of Supercritical Fluids, 25, 197-212. http://dx.doi.org/10.1016/S0896-8446(02)00142-0

[2] Cygnarowicz, M.L., Maxwell, R.J. and Seider, W.D. (1990) Equilibrium Solubilities of $\beta$-Carotene in Supercritical Carbon Dioxide. Fluid Phase Equilibria, 59, 57-71. http://dx.doi.org/10.1016/0378-3812(90)85146-2

[3] Ashour, I., Almehaideb, R., Fateen, S.-E. and Aly, G. (2000) Representation of Solid-Supercritical Fluid Phase Equilibria Using Cubic Equations of State. Fluid Phase Equilibria, 167, 41-61. http://dx.doi.org/10.1016/S0378-3812(99)00314-3

[4] Tabernero, A., del Valle, E.M.M. and Galán, MÁ. (2010) A Comparison between Semiempirical Equations to Predict the Solubility of Pharmaceutical Compounds in Supercritical Carbon Dioxide. The Journal of Supercritical Fluids, 52, 161-174. http://dx.doi.org/10.1016/j.supflu.2010.01.009

[5] Cortesi, A., Kikic, I., Alessi, P., Turtoi, G. and Garnier, S. (1999) Effect of Chemical Structure on the Solubility of Antioxidants in Supercritical Carbon Dioxide: Experimental Data and Correlation. The Journal of Supercritical Fluids, 4, 139-144. http://dx.doi.org/10.1016/S0896-8446(98)00119-3

[6] Garnier, S., Neau, E., Alessi, P., Cortesi, A. and Kikic, I. (1999) Modeling Solubility of Solids in Supercritical Fluids Using Fusion Properties. Fluid Phase Equilibria, 158-160, 491-500. http://dx.doi.org/10.1016/S0378-3812(99)00151-X

[7] Méndez-Santiago, J. and Teja, A.S. (1999) The Solubility of Solids in Supercritical Fluids. Fluid Phase Equilibria, 158160, 501-510.http://dx.doi.org/10.1016/S0378-3812(99)00154-5

[8] Harvey, A.H. (1990) Supercritical Solubility of Solids from Near-Critical Dilute-Mixture Theory. The Journal of Phy- 
sical Chemistry, 94, 8403-8406. http://dx.doi.org/10.1021/j100385a009

[9] Nasri, L., Bensaad, S. and Bensetiti, Z. (2013) Correlation and Prediction of the Solubility of Solid Solutes in Chemically Diverse Supercritical Fluids Based on the Expanded Liquid Theory. Advances in Chemical Engineering and Science, 3, 255-273. http://dx.doi.org/10.4236/aces.2013.34033

[10] Kumar, S.K. and Johnston, K.P. (1988) Modeling the Solubility of Solids in Supercritical Fluids with Density as the Independent Variable. The Journal of Supercritical Fluids, 1, 15-22. http://dx.doi.org/10.1016/0896-8446(88)90005-8

[11] Johanna, M.H. and Sengers, L. (1991) Solubility near the Solvent's Critical Point. The Journal of Supercritical Fluids, 4, 215-222. http://dx.doi.org/10.1016/0896-8446(91)90013-V

[12] Sparks, D.L., Hernandez, R. and Estévez, L.A. (2008) Evaluation of Density-Based Models for the Solubility of Solids in Supercritical Carbon Dioxide and Formulation of a New Model. Chemical Engineering Science, 63, 4292-4301. http://dx.doi.org/10.1016/j.ces.2008.05.031

[13] Goldfarb, J.L. and Suuberg, E. M. (2008) Vapor Pressures and Enthalpies of Sublimation of Ten Polycyclic Aromatic Hydrocarbons Determined via the Knudsen Effusion Method. Journal of Chemical \& Engineering Data, 53, 670-676. http://dx.doi.org/10.1021/je7005133

[14] Prausnitz, J.M., Lichtenthaler, R.N., de Azevedo, E.G. (1999) Molecular Thermodynamics of Fluid-Phase Equilibria. 3rd Edition, Prentice Hall Inc., Engelwood Cliffs.

[15] Schmitt, W.J. and Reid, R.C. (1986) Solubility of Monofunctional Organic Solids in Chemically Diverse Supercritical Fluids. Journal of Chemical \& Engineering Data, 31, 204-212. http://dx.doi.org/10.1021/je00044a021

[16] McEachern, D.M., Sandoval, O. and Iniguez, J.C. (1975) Vapor Pressures, Derived Enthalpies of Sublimation, Enthalpies of Fusion, and Resonance Energies of Acridine and Phenazine, The Journal of Chemical Thermodynamics, 7, 299306. http://dx.doi.org/10.1016/0021-9614(75)90069-5

[17] Delle, S.A. (1997) The Vapor Pressure of Environmentally Significant Organic Chemicals: A Review of Methods and Data at Ambient Temperature. Journal of Physical and Chemical Reference Data, 26, 157-193. http://dx.doi.org/10.1063/1.556006

[18] Hansen, P.C. and Eckert, C.A. (1986) An Improved Transpiration Method for the Measurement of Very Low Vapor Pressure. Journal of Chemical \& Engineering Data, 31, 1-3. http://dx.doi.org/10.1021/je00043a001

[19] Oliveira, J.A.S.A., Monte, M.J.S., Notario, R. and Ribeiro da Silva, M.D.M.C. (2014) Experimental and Computational Study of the Thermodynamic Properties. The Journal of Chemical Thermodynamics, 76, 56-63. http://dx.doi.org/10.1016/j.jct.2014.03.005

[20] Huang, Z.S., Kawi, S. and Chiew, Y.C. (2004) Application of the Perturbed Lennard-Jones Chain Equation of State to Solute Solubility in Supercritical Carbon Dioxide. Fluid Phase Equilibria, 216, 111-122. http://dx.doi.org/10.1016/j.fluid.2003.10.004

[21] Bardi, G., Malaspina, L. and Piacenti, V. (1973) Vapor Pressure and Sublimation Enthalpy of Anthraquinone and of 1,5- and 1,8-Dihydroxyanthraquinone. Journal of Chemical \& Engineering Data, 18, 126-130. http://dx.doi.org/10.1021/je60057a024

[22] Sasse, K., N’guimbi, J., Jose, J. and Merlin, J.C. (1989) Tension de vapeur d'hydrocarbures polyaromatiques dans le domaine $10^{-3}-10$ Torr. Thermochimica Acta, 146, 53-61. http://dx.doi.org/10.1016/0040-6031(89)87075-3

[23] Da Silva, M.A.V. and Ribeiro, Monte, M.J.S. (1990) The Construction, Testing and Use of a New Knudsen Effusion Apparatus. Thermochimica Acta, 171, 169-183. http://dx.doi.org/10.1016/0040-6031(90)87017-7

[24] Eckert, Z. (1983) Correlation and Prediction of Solid-Supercritical Fluid Phase Equilibria. Industrial and Engineering Chemistry Process Design and Development, 22, 582-588. http://dx.doi.org/10.1021/i200023a005

[25] Colomina, M., Jimenez, P., Roux, M.V. and Turrion, C. (1979) Thermochemical Properties of Naphthalene Derivatives. V. Formation Enthalpies of 2,3-Dimethylnaphthalene. Anales de Química, 75, 620-624.

[26] Stephenson, R.M. and Malanowski, S. (1987) Handbook of the Thermodynamics of Organic Compounds. Elsevier, New York. http://dx.doi.org/10.1007/978-94-009-3173-2

[27] Lide, D.R. (2001) CRC Handbook of Chemistry and Physics. 84th Edition, CRC Press, Boca Raton.

[28] Baccanari, D.P., Novinski, J.A., Pan, Y.-C., Yevitz, M.M. and Swain, H.A. (1968) Heats of Sublimation and Vaporization at $25^{\circ}$ of Long Chain Fatty Acids and Methyl Esters. Transactions of the Faraday Society, 64, 1201-1205. http://dx.doi.org/10.1039/tf9686401201

[29] Welsh, W.J., Tong, W., Collantes, E.R., Chickos, J.S. and Gagarin, S.G. (1997) Enthalpies of Sublimation and Formation of Polycyclic Aromatic Hydrocarbons (PAHs) Derived from Comparative Molecular Field Analysis (CoMFA): Application of Moment of Inertia for Molecular Alignment. Thermochimica Acta, 290, 55-64. http://dx.doi.org/10.1016/S0040-6031(96)03048-1 
[30] Arshadi, M.R. (1974) Determination of Heats of Sublimation of Organic Compounds by a Mass Spectrometric-Knudsen Effusion Method. Journal of the Chemical Society, Faraday Transactions, 70, 1569-1571. http://dx.doi.org/10.1039/f19747001569

[31] Colomina, M., Roux, M.V. and Turrion, C. (1974) Thermochemical Properties of Naphthalene Compounds. II. Enthalpies of Combustion and Formation of the 1- and 2-Naphthols. The Journal of Chemical Thermodynamics, 6, 571-576. http://dx.doi.org/10.1016/0021-9614(74)90044-5

[32] Huang, C.-C., Tang, M., Tao, W.-H. and Chen, Y.-P. (2001) Calculation of the Solid Solubilities in Supercritical Carbon Dioxide Using a Modified Mixing Model. Fluid Phase Equilibria, 179, 67-84. http://dx.doi.org/10.1016/S0378-3812(00)00483-0

[33] Balson, E.W. (1947) Studies in Vapor Pressure Measurement, Part III.—An Effusion Manometer Sensitive to $5 \times 10^{-6}$ Millimeters of Mercury: Vapor Pressure of D.D.T. and Other Slightly Volatile Substances. Transactions of the Faraday Society, 43, 54-60. http://dx.doi.org/10.1039/tf9474300054

[34] Oja, V. and Suuberg, E. M. (1998) Vapor Pressures and Enthalpies of Sublimation of Polycyclic Aromatic Hydrocarbons and Their Derivatives. Journal of Chemical \& Engineering Data, 43, 486-492. http://dx.doi.org/10.1021/je970222l

[35] Malaspina, L., Bardi, G. and Gigli, R. (1974) Simultaneous Determination by knudsen-Effusion Microcalorimetric Technique of the Vapor Pressure and Enthalpy of Vaporization of Pyrene and 1,3,5-Triphenylbenzene. The Journal of Chemical Thermodynamics, 4, 1053-1064. http://dx.doi.org/10.1016/0021-9614(74)90067-6

[36] Tochigi, K., Iizumi, T., Sekikawa, H., Kurihara, K. and Kojima, K. (1998) High-Pressure Vapor-Liquid and Solid-Gas Equilibria Using a Peng-Robinson Group Contribution Method. Industrial \& Engineering Chemistry Research, 37, 37313740. http://dx.doi.org/10.1021/ie970060m

[37] Chickos, J.S. and Acree Jr., W.E. (2002) Enthalpies of Sublimation of Organic and Organometallic Compounds.19102001. Journal of Physical and Chemical Reference Data, 31, 537-698. http://dx.doi.org/10.1063/1.1475333

[38] Nass, K., Lenoir, D. and Kettrup, A. (1995) Calculation of the Thermodynamic Properties of Polycyclic Aromatic Hydrocarbons by an Incremental Procedure. Angewandte Chemie International Edition in English, 34, 1735-1736. http://dx.doi.org/10.1002/anie.199517351

[39] Hansen, B.N., Harvey, A.H., Coelho, J.A.P., Palavra, A.M.F. and Bruno, T.J. (2001) Solubility of Capsaicin and $\beta$ Carotene in Supercritical Carbon Dioxide and in Halocarbons. Journal of Chemical \& Engineering Data, 46, 10541058. http://dx.doi.org/10.1021/je000255s 